\title{
Using learned under-sampling pattern for increasing speed of cardiac cine MRI based on compressive sensing principles
}

\author{
Pooria Zamani ${ }^{1}$, Mohammad Kayvanrad ${ }^{2}$ and Hamid Soltanian-Zadeh ${ }^{1,3,4,5^{*}}$
}

\begin{abstract}
This article presents a compressive sensing approach for reducing data acquisition time in cardiac cine magnetic resonance imaging (MRI). In cardiac cine MRI, several images are acquired throughout the cardiac cycle, each of which is reconstructed from the raw data acquired in the Fourier transform domain, traditionally called k-space. In the proposed approach, a majority, e.g., $62.5 \%$, of the k-space lines (trajectories) are acquired at the odd time points and a minority, e.g., 37.5\%, of the k-space lines are acquired at the even time points of the cardiac cycle. Optimal data acquisition at the even time points is learned from the data acquired at the odd time points. To this end, statistical features of the k-space data at the odd time points are clustered by fuzzy c-means and the results are considered as the states of Markov chains. The resulting data is used to train hidden Markov models and find their transition matrices. Then, the trajectories corresponding to transition matrices far from an identity matrix are selected for data acquisition. At the end, an iterative thresholding algorithm is used to reconstruct the images from the under-sampled k-space datasets. The proposed approaches for selecting the k-space trajectories and reconstructing the images generate more accurate images compared to alternative methods. The proposed undersampling approach achieves an acceleration factor of 2 for cardiac cine MRI.
\end{abstract}

Keywords: cardiac cine MRI, fuzzy c-means, hidden Markov model, compressive sensing

\section{Introduction}

Some time-consuming applications of magnetic resonance imaging (MRI), especially dynamic ones, such as cardiac, functional magnetic resonance imaging (fMRI), diffusion tensor imaging, and spectroscopic imaging are developed in recent years. Consequently, it seems inevitable to find some ways to increase MRI speed without losing image quality. Reducing MRI data acquisition time may increase patient's comfort and economic efficiency but decrease spatial or temporal resolution of images. The hardware solution that reduces MRI data acquisition time by using more powerful gradient amplifiers is limited by technical and biological considerations like nerve stimulation. Therefore, noticeable effort has been put into developing methods to use intrinsic redundancy and

\footnotetext{
* Correspondence: hszadeh@ut.ac.ir

${ }^{1}$ Control and Intelligent Processing Center of Excellence (CIPCE), School of Electrical and Computer Engineering, University of Tehran, Tehran 1439957131, Iran

Full list of author information is available at the end of the article
}

correlation of MRI data in the $k-t$ space to reconstruct images without a complete sampling of the $k-t$ space. We can divide these approaches into the following three categories [1]:

1. Exploiting correlations in $k$-space, such as parallel imaging [2,3];

2. Exploiting temporal correlations, such as fMRI example of UNFOLD [4];

3. Exploiting correlations in both $k$-space and time domain, such as $k$-t BLAST [1].

Although the above methods are widely used to speed up MRI, they have limitations like decreasing SNR, being exclusively applicable to dynamic MRI, and need for special hardware or training data.

In recent years, compressive sensing (CS) theory $[5,6]$ has been used to recover sparse signals from undersampled data. Applying this general signal processing principle to MRI requires that [7]: (a) the desired image 
has a sparse representation, i.e., it is compressible, in a known transform domain, e.g., wavelets; (b) the aliasing artifacts due to $k$-space undersampling are incoherent (noise like) in that transform domain. Random sampling in $k$-space has the best incoherency with the basis of sparsifying transform but this can be challenging in MRI undersampling. Random undersampling of $k$-space, including random sampling in both of the phase encoding $(\mathrm{PE})$ and frequency encoding directions, can be as timeconsuming as full sampling of $k$-space because of MRI principles. We will address this problem in the article; (c) a nonlinear reconstruction is used to enforce both sparsity of the image representation and consistency with the acquired data. Fortunately, there are efficient algorithms to solve underdetermined systems of linear equations with respect to the sparsity constraint corresponding to condition (c) [8]. We will use an iterative thresholding method to reconstruct undersampled sparse signals.

$\mathrm{CS}$ is robust to noise and does not require any special hardware. Thus, using CS principles to speed up MRI can decrease the above-mentioned drawbacks. Successful results have been achieved to speed up MRI by applying CS to MRI [7,9-15]. Some of them focused on the first stage, i.e., determining undersampling pattern in the $k-t$ space and others dealt with reconstruction algorithms.

In this article, we will consider both topics for cardiac cine MRI. First, we will present a solution to sample some $k$-space trajectories. To this end, we fill the upper half of $k$-space completely for odd time points in a cardiac cycle. Then, we process them to determine trajectories to sample using fuzzy $c$-means and hidden Markov model (HMM) principles. Then, we undersample $k$-spaces corresponding to the even time points in a cardiac cycle, according to the above results. We use an iterative method to reconstruct desired images from the undersampled data by sparsity constraint in both of the above-mentioned steps.

The proposed technique is a practical method for applying CS to MRI, benefiting from MRI specifications such as Hermitian symmetry of $k$-space and using available data to determine how we can undersample them.

In the following section, we describe the concepts used in this article such as CS [5,6], HMM, and the iterative thresholding algorithm for sparse recovery $[16,17]$. Also, we present details of the proposed method. Using fuzzy clustering, we study $k$-space trajectories through HMM and then select the ones that have largest variations. Afterwards, we reconstruct the desired images by going through a sequence of thresholding iterations. In the results section, we present experimental results for an application of the proposed method to reconstruct undersampled cardiac cine MRI data. Then, we present strengths and drawbacks of the proposed method and some suggestions to improve it. In the conclusions section, we list the main findings of our research. Finally, we show that our method results in improved quality of reconstruction in comparison with some other methods.

\section{Main text}

In this section, we introduce the theory of CS $[5,6,18]$ and then discuss training of the HMM [19] based on some observations. Next, we explain the iterative thresholding algorithm used for reconstruction from undersampled $k$-space data. At the end, we describe the details of our proposed algorithm.

\section{Compressive sensing}

Let $x \in R^{N}$, and the matrix $\Lambda=\left[\lambda_{1}, \lambda_{2}, \ldots, \lambda_{N}\right]$ be a basis for $R^{N}$. We say that $x$ is $K$-sparse if we have

$$
x=\sum_{i=1}^{K} \theta_{i} \lambda_{i}
$$

and $K \ll N$. Consider also an $M \times N$ measurement matrix $\Phi, M \ll N$, where the rows of $\Phi$ are incoherent with the columns of $\Lambda$. Incoherency between rows of the measurement matrix and the basis vectors means that we need all of the vectors in the second set to expand each of the vectors in the first set and vice versa. CS theory states that only $m(m)$ is of $O(K \cdot \log (N))$ incoherent measurements $y=\Phi x$, are required to reconstruct the signal, $x$, with a high probability.

To reconstruct the desired signal, $x$, we should solve the following combinatorial optimization problem

$$
\left\{\begin{array}{c}
\min .\|\theta\|_{0} \\
\text { s.t. }\|\Phi \mathrm{x}-\mathrm{y}\|^{2} \leq \varepsilon
\end{array}\right.
$$

where is the number of nonzero coefficients of the transformed version of $x$ in the domain which is sparse. Solving this non-convex problem is very computationally expensive and sometimes infeasible. Thus, one can equivalently solve the convex $l_{1}$ minimization:

$$
\left\{\begin{array}{c}
\min .\|\theta\|_{1} \\
\text { s.t. }\|\Phi x-y\|^{2} \leq \varepsilon
\end{array}\right.
$$

The threshold parameter $\epsilon$ is usually set below the expected noise level [7]. Necessary conditions for equivalency between (2) and (3) are beyond the scope of this article but in brief, we can state that it requires: (a) sparsity of the desired signal, $x$; and (b) some conditions on the measurement matrix, $\Phi$. Details of these conditions are presented in [5,6]. There are some efficient algorithms to solve it [8]. We will use an iterative thresholding method for this purpose.

Incoherence is an essential condition for this theory to be successful. For example, if the rows of the measurement matrix are i.i.d. Gaussian random vectors, such a matrix is incoherent with any other fixed matrix with a high probability. The problem of using CS in MRI is 
exactly lying here, because random sampling of $k$-tspace can be as time-consuming as complete sampling.

\section{Hidden Markov models}

To explain HMMs [19], we should describe Markov models first. A Markov process is a time-varying random phenomenon for which the Markov property holds. We will mathematically define the Markov property but in words, a stochastic process with the Markov property, or memorylessness, is one for which conditional on the present state of the system, its future and past are independent. The term Markov chain is used to mean a Markov process which has a discrete (finite or countable) state-space. Suppose that for a Markov model, we have

- Observable states: $1,2, \ldots, N$.

- Observed sequence: $q_{1}, q_{2}, \ldots, q_{t}, \ldots, q_{T}$

First-order Markov property means:

$$
P\left(q_{t}=j \mid q_{t-1}=i, q_{t-2}=k, \ldots\right)=P\left(q_{t}=j \mid q_{t-1}=i\right)
$$

A Markov model can be recognized by its state transition matrix $T$ :

$$
\left[t_{i j}\right]
$$

where

$$
t_{i j}=P\left(q_{t}=j \mid q_{t-1}=i\right) \quad 1 \leq i, j \leq N N
$$

with the constraints

$$
\left\{\begin{array}{c}
t_{i j} \geq 0, \quad \forall i, j \\
\sum_{j=1}^{N} t_{i j}=1, \quad \forall i
\end{array}\right.
$$

An HMM is a statistical Markov model in which the system being modelled is assumed to be a Markov process with unobserved (hidden) states. In a regular Markov model, the state is directly visible to the observer and therefore the state transition probabilities are the only parameters. In an HMM, the state is not directly visible but the output that depends on the state is visible. Each state has a probability distribution over the possible output tokens. Therefore, the sequence of tokens generated by an HMM gives some information about the sequence of states. Note that the adjective 'hidden' refers to the state sequence through which the model passes, not to the parameters of the model; even if the model parameters are known exactly, the model is still 'hidden'.

Suppose for an HMM, we have

- Set of states: $1,2, \ldots, N$.

- Set of outputs: $1,2, \ldots, M$.
- state transition matrix $T$ :

$\left[t_{i j}\right]$

where

$$
t_{i j}=P\left(q_{t}=j \mid q_{t-1}=i\right) \quad 1 \leq i, j \leq N N
$$

- Observation probability distribution:

$$
B_{j}(k)=P\left(o_{t}=k \mid q_{t}=j\right) \quad 1 \leq k \leq M, 1 \leq j \leq N
$$

- $\pi$, the initial state distribution:

$$
\pi_{i}=P\left(q_{1}=i\right) \quad 1 \leq i \leq N
$$

An HMM can be described by the last three parameters: $\lambda=(T, B, \pi)$.

In this article, we employ HMM to study temporal behavior of $k$-space lines. To this end, we consider outputs of clustering step as the set of outputs of a HMM $(O)$. We want to estimate $\lambda$ such that $P(O \mid \lambda)$ is maximized. Fortunately, there is Baum-Welch algorithm to solve this problem. As explained later, we use the estimated $T$ to decide about including or not including a $k$ space line in the sampling pattern.

\section{Iterative thresholding reconstruction}

We will use the iterative thresholding reconstruction algorithm described in $[16,17]$ for image reconstruction from undersampled $k$-space data. The Landweber thresholding algorithm was originally presented in [20]. For the sake of completeness, we present a brief description of the reconstruction algorithm here.

In a discrete setting, assume an $N \times N$ image, $f(x, y)$, and its discrete Fourier transform, $F(u, v)=\mp\{f(x, y)\}$, where $F$ denotes the Fourier transform. Define a sampling mask, $S$, acting on the Fourier transform of the image, $F(u, v)$,

$$
S(u, v)=\left\{\begin{array}{lc}
1 & F(u, v) \text { sampled } \\
0 & F(u, v) \text { not sampled }
\end{array}\right.
$$

The relationship between the incomplete set of observed samples, $G$, and the image, $F$, can be expressed by the above sampling mask as follows:

$$
G=S \cdot F
$$

where - denotes the point-wise product of $S$ and $F$.

Clearly, the objective is to reconstruct the image, $f$, from the available Fourier domain observations, $G$, while the sampling mask, $S$, is also known. The reconstruction algorithm can be summarized as below: 
1. initialization (min. energy reconstruction): $n=0, F^{n}=$ $F^{0}=S \cdot G$

2. $f^{n}=\left|\mathrm{F}^{-1}\left\{F^{n}\right\}\right|$

3. $n++$

4. maintain the constraint: $h^{n}=S_{\gamma}\left(\rho^{n-1}\right)$

5. recovery of the original observations: $F^{n}=G+H^{n}$ $S \cdot H^{n}$

6. convergence? Yes: exit; no: go to 2

Here, superscripts denote the iteration number. $S \gamma$ denotes soft thresholding operation defined as:

$$
S_{\gamma}(g)=\Sigma_{i} S_{\gamma}\left(\left|g, \psi_{i}\right\rangle\right) \psi_{i}
$$

where

$$
S_{\gamma}(x)= \begin{cases}x+\frac{\gamma}{2}, & x \leq-\frac{\gamma}{2} \\ 0, & |x| \leq \frac{\gamma}{2} \\ x-\frac{\gamma}{2}, & x \geq \frac{\gamma}{2}\end{cases}
$$

and $\left\{\psi_{i}\right\}$ is a set of orthonormal basis functions for the sparse domain. Thresholding, $S_{\gamma}$ is essentially a sparsitypromoting operation. In brief, this operation promotes sparsity in each iteration.

\section{Proposed algorithm}

As mentioned, increasing MRI speed by undersampling $k$ - $t$-space may decrease SNR of the reconstructed images. Similarly, the MRI acquisition time may be decreased by acquiring undersampled $k$-space data. This, however, may result in loss of resolution or aliasing reconstruction artifact in the spatial domain. Nevertheless, MR images often have sparse or compressible representations in appropriate transform domains such as wavelets. In fact, one may be able to use this sparsity as an a priori reconstruction constraint to interpolate missing $k$-space data. Also, use of CS in MRI does not need any specific hardware changes. Therefore, in this research, we focus on increasing MRI speed by CS.

To this end, we address two problems involved in applying CS to MRI: (1) how to choose the undersampling pattern of $k$-space; and (2) how to reconstruct an image from the undersampled data. We use the data, itself, to learn the undersampling pattern and apply an iterative thresholding method for reconstruction from undersampled $k$-space data.

Our proposed method is expressed in the following main stages and the flowchart in Figure 1 illustrates its steps.

For the odd points in the cardiac cycle,

- Sample the upper half of $k$-space.

- Reconstruct the images corresponding to each coil channel.
- Process the available $k$-space trajectories to specify the ones that should be sampled.

In the above steps, we gather and use $k$-space of odd time points to reconstruct the corresponding images and determine sampling pattern of even time points.

For the even time points in the cardiac cycle,

- Sample specific $k$-space trajectories according to the results of the proceeding step.

- Reconstruct images corresponding to each coil channel.

To clarify details of the mentioned stages, we should note the following.

- We sample the central lines in addition to the upper half of $k$-space. The resulting mask covers $62.5 \%$ of all lines. According to the MRI techniques, $5 / 8$ or $62.5 \%$ of all lines is a standard fraction for half scan.

- We reconstruct images using undersampled $k$ space data by iterative thresholding, as described before.

- Images corresponding to the multi-channel coil are computed using the images of each coil channel and sum-of-squares rule [21,22].

- $K$-space corresponding to each image is obtained by $2 \mathrm{D}$ Fourier transform.

As stated previously, we study temporal behavior of $k$ space patterns to decide about selecting or not selecting them in the sampling pattern. To this end, we employ fuzzy $c$-means clustering and HMM's as intermediate tools. We cluster features extracted from each $k$-space lines and then consider these class labels as states of some HMM's. Finally, we estimate state transition matrices of these HMM's and use them to decide about selecting or not selecting specific $k$-space lines in the sampling pattern.

The processing stage is described below.

- Feature extraction of $k$-space patterns: Mean, standard deviation, maximum, and median of each trajectory are extracted.

- Clustering the extracted features: Clustering method is fuzzy $c$-means and the number of clusters is about $1 / 4$ of the available trajectories. Clustering is done for the available trajectories of each coil channel. It is possible to accelerate dynamic MRI, 2 to 8 times dependent on the type of MRI [4]. Thus, we assume that every 4 (between 2 and 8 ) $k$-space lines in the time direction, can be clustered into one cluster. We choose fuzziness factor equals to 1.125. 

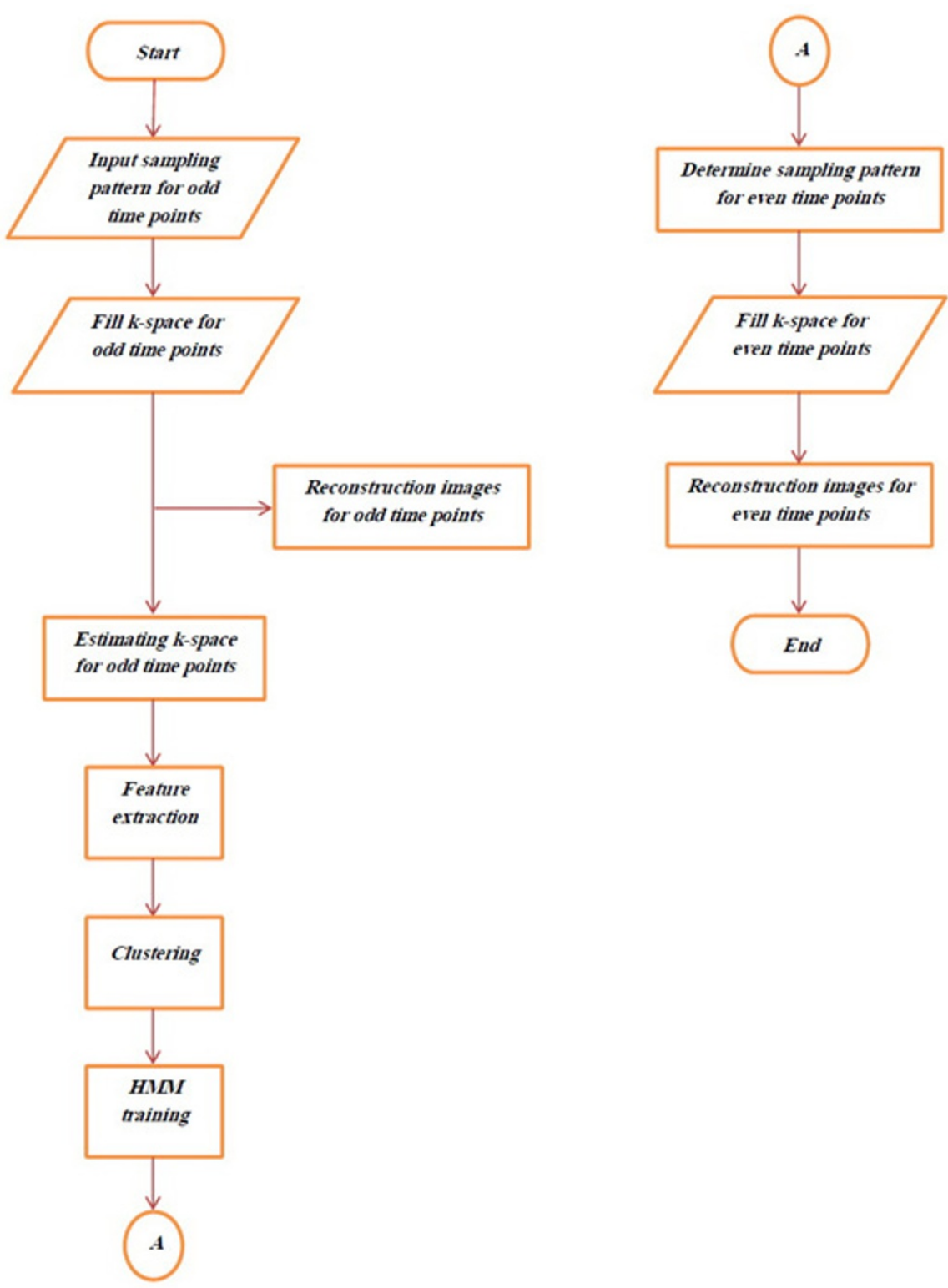

Figure 1 Flowchart of the proposed algorithm. Different steps of the proposed algorithm and their connections.

- Class label rearrangement: After clustering, the resulting labels for each coil channel may be different. Thus, we have to rearrange these labels such that the clusters with similar centers have the same label. Class label rearrangement is automatic. In the fuzzy clustering section, we first apply fuzzy clustering to the extracted features from $k$-space corresponding to the entire coil. In this way, we obtain cluster centers and class labels for each $k$-space line. After that, we apply fuzzy clustering to $k$-space of each coil channel. Clusters of the entire coil and clusters of each coil channel should have same labels if they have the nearest centers. We compare cluster centers of coil channels with cluster centers of the entire coil and change them according to the above rule, if necessary. - HMM training: The above clusters are assumed as outputs $(O)$ of Markov models. We estimate parameters of HMM, $\lambda=(T, B, \pi)$, such that a posteriori probability of outputs, $P(O \mid \lambda)$, is maximized. This is done using Baum-Welch algorithm. We use the resulting transition matrices to decide about selecting or not selecting each $k$-space line.

- Selecting or not selecting a $k$-space trajectory (line) to be sampled in the even points of the cardiac cycle: A steady Markov chain has a transition matrix near an identity matrix. Thus, we select the trajectories 
that have transition matrices different from an identity matrix. Such trajectories are expected to have more changes than the others and are more useful for improving the reconstruction quality.

\section{Results}

\section{Pre-processing}

True image of each coil channel and computed image of the entire coil, resulting from 2D inverse Fourier transform of fully sampled $k$-space of time point 1 , are shown in Figure 2. The corresponding images, generated by the proposed iterative reconstruction method, are shown in Figure 3. The undersampling mask includes the upper $62.5 \%$ of $k$-space lines along the PE direction as shown in Figure 4a. It is clear that the quality of the reconstructed images is lower than those of the true images.

\section{Clustering}

As described in the previous sections, we extract features of each $k$-space line and then apply clustering to them. The clustering results are shown in Figure 5 for four samples of $k$-space trajectories.

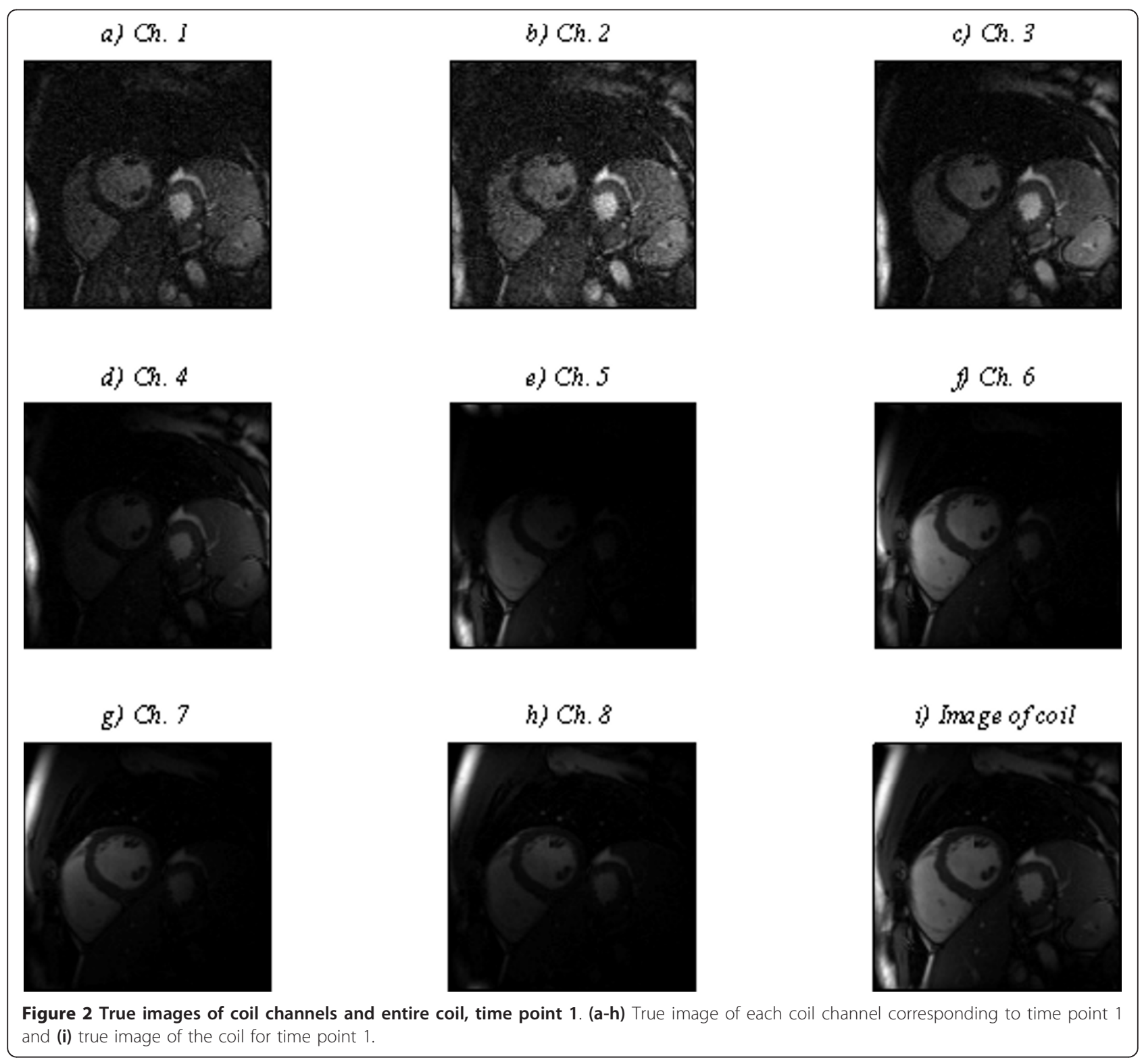




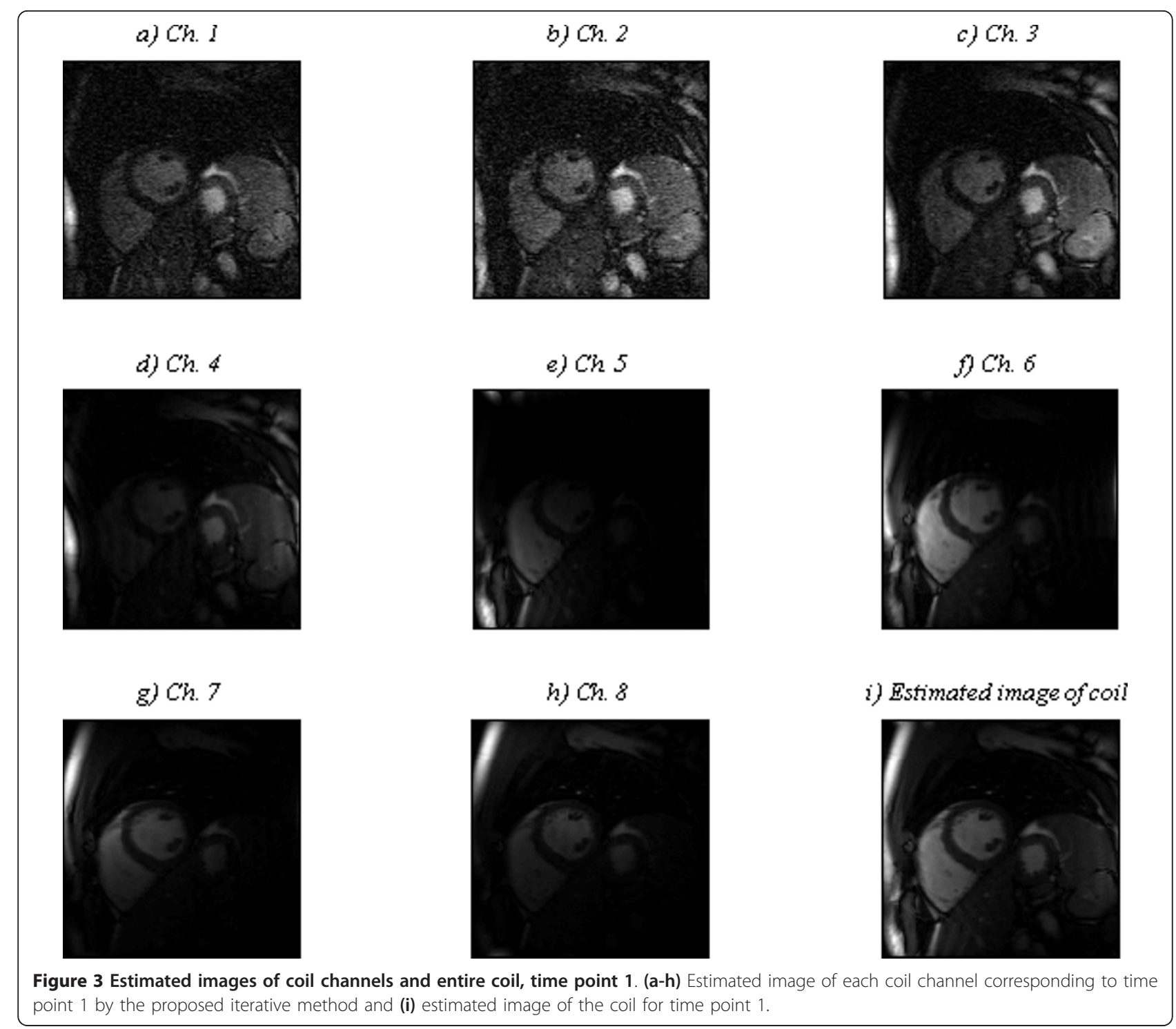

\section{HMM training}

We expect that the trajectories that include a large amount of information also have more changes in the related class labels than the others. The results of training HMM's are shown in Figure 6. We used Baum-Welch algorithm to train HMM's. The inputs of the training stage are the cluster labels generated in the clustering step and the outputs are the transition matrices related to $k$-space trajectories. As described before, we hypothesize that the trajectories that have transition matrices far from the identity matrix have more information than the others and should be more useful for improving the reconstruction quality. Thus, we sort $k$-space trajectories based on their differences from the identity matrix. Then, we compose the undersampling mask by selecting the trajectories that are on the top of the list. We use this mask, shown in Figure 4b, to undersample $k$-space in the even time points of the cardiac cycle.

\section{Reconstruction}

In the proposed algorithm, we first extract a mask for undersampling even time points of cardiac cycle. After that, we reconstruct images of even time points using our iterative method. Thus, to show the performance of the proposed algorithm, we first reconstruct cardiac images for even time points by the extracted mask and some other sample masks, as shown in Figure 4c-f. The results for a sample even time point, point 18 , are shown in Figure 7 . Second, we use $62.5 \%$ of upper mask for odd time points and the extracted mask for even ones and apply our proposed iterative, Lustig's nonlinear conjugate gradient [7], and $k-t$ FOCUSS method [14] to reconstruct 


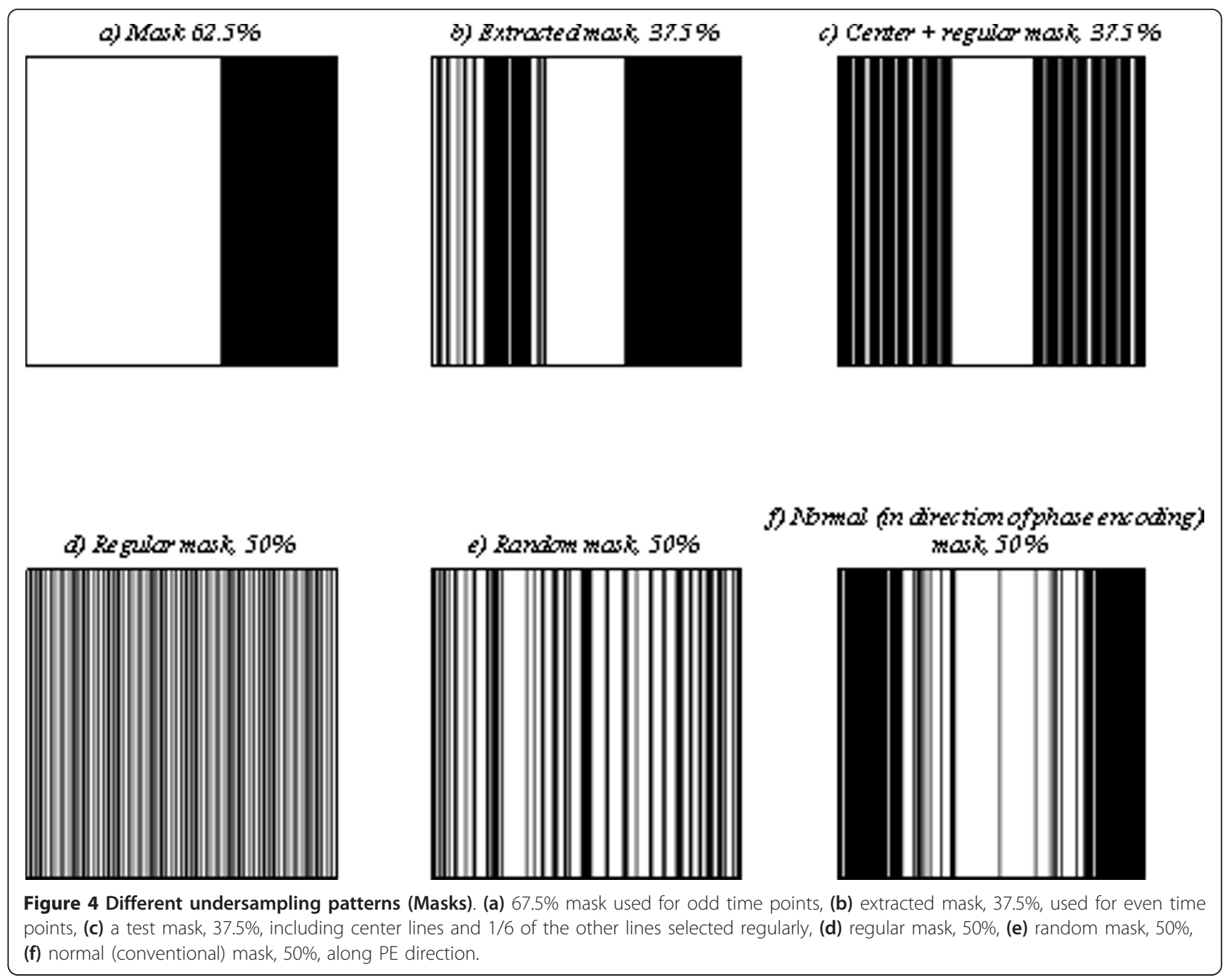

images for all time points. The results for a sample even time point, point 18, are shown in Figure 8.

In addition, we compute all cardiac images using SENSE [3] and GRAPPA [23] and 50\% regular mask, which are the main methods in parallel MRI. We employ conventional minimum energy and Hemodyne filtering [22], a method based on Hermitian symmetry, and $50 \%$ upper mask to reconstruct undersampled cardiac images, too. The results for a sample even time point, point 18, are shown in Figure 9.

\section{Discussion}

We have proposed a method to obtain $62.5 \%$ of $k$-space data at odd time frames and estimate the sampling trajectory at even time frames. In ECG-gated cardiac MRI, $k$-space data for each cardiac phase (odd and even time frames) are obtained by collecting $k$-space data across several heart beats. Since even and odd time frames from a prospective gating are synthesized ones, they are not obtained in sequential order. Thus, the proposed algorithm may seem infeasible. However, it should be noted that when we do not fill $k$-space for even time points, we use the time to acquire more $k$-space data of the odd time points. Therefore, the number of heart bits (acquisition time) required for sampling of $k$-spaces of odd time points reduces. The acquisition time of even time frames, which is done afterwards, is reduced in the same way. Thus, the proposed method decreases the total time required for data acquisition. Details are presented in Additional file 1.

The acceleration factor of 2 demonstrated in this study may not look impressive for dynamic MRI as some existing methods of fast MRI, such as parallel imaging, may reach the same acceleration factor. As such, why should one use the proposed method to get the same acceleration factor? The answer is that this article proposes a novel approach to use the data, itself, and determine an optimal sampling pattern. In addition, the reconstruction 

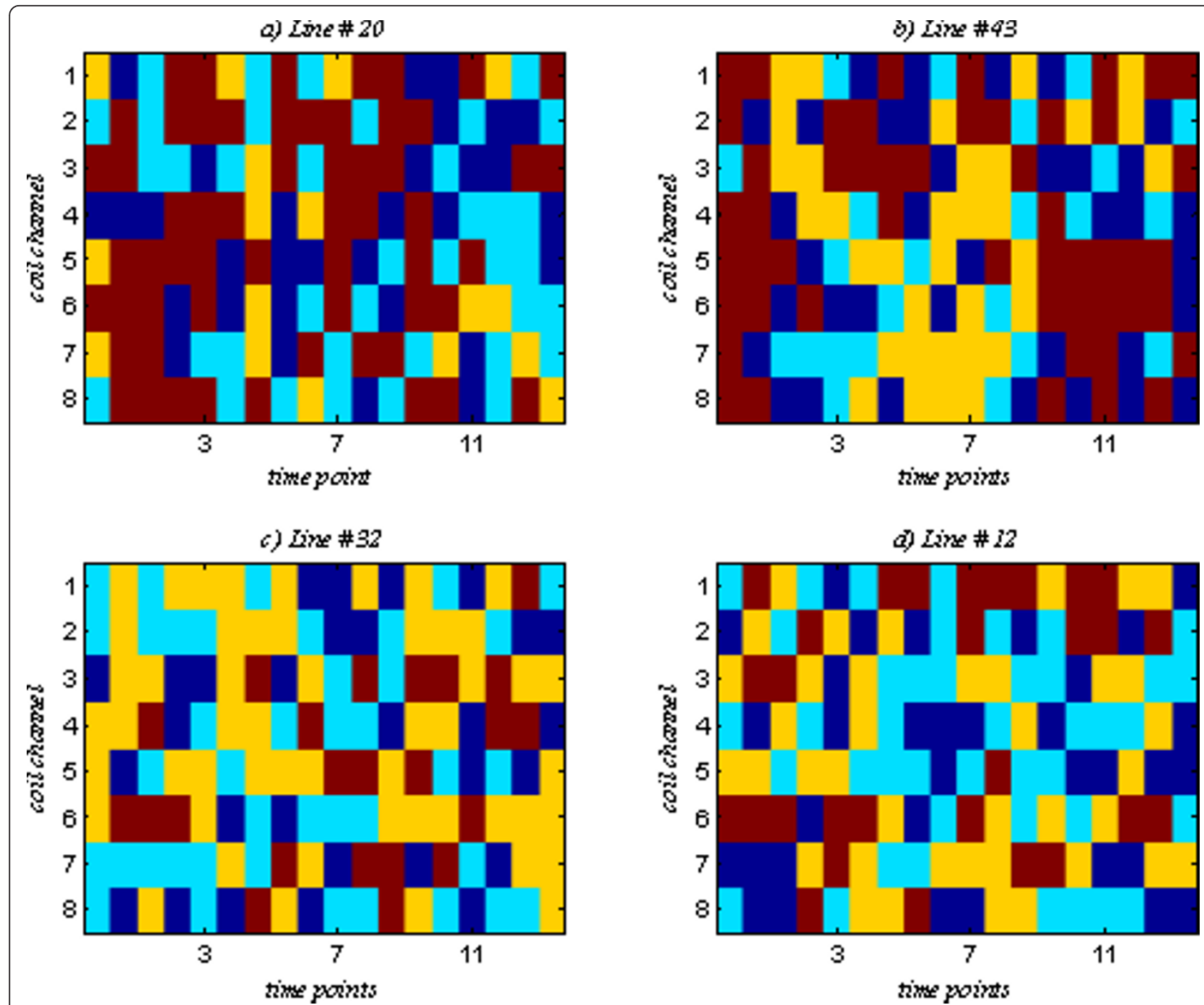

Figure $\mathbf{5}$ Sample results of clustering. $(\mathbf{a}, \mathbf{b})$ Clustering results for two sample $k$-space lines. These two lines were selected for sampling mask. (c, d) Clustering results for two sample $k$-space lines. These two lines were not selected for sampling mask. The vertical axis refers to eight coil channels and the horizontal one shows odd time points.

error of the proposed method is lower than those of the other methods. Moreover, the method is developed for the Cartesian sampling pattern. Other sampling patterns may lead to higher acceleration factors.

Increasing the percentage of the selected lines reduces reconstruction error and acceleration factor. To this end, the number of clusters must be selected according to the possible acceleration rates in dynamic MRI. Finally, reducing the error bound is expected to reduce the reconstruction error but will increase the reconstruction time.

\section{Strengths}

We divide the data acquisition process into two steps: data gathering for odd and even time points of the cardiac cycle. This allows our method to use available data to optimize the undersampling pattern and enhance the reconstruction results.

We use an intrinsic property of MRI, Hermitian symmetry of $k$-space data, and employ Fuzzy $c$-means and HMM's as intermediate tools to study time variations of $k$-space trajectories. Our reconstruction method has less error than some others state-of-theart methods, is robust to noise, and runs relatively fast. The proposed method does not require any hardware changes and thus it can be applied using the present scanners.

\section{Drawbacks}

Our method is only applicable to dynamic MRI methods. We have to study time variations of a part of $k$ - 


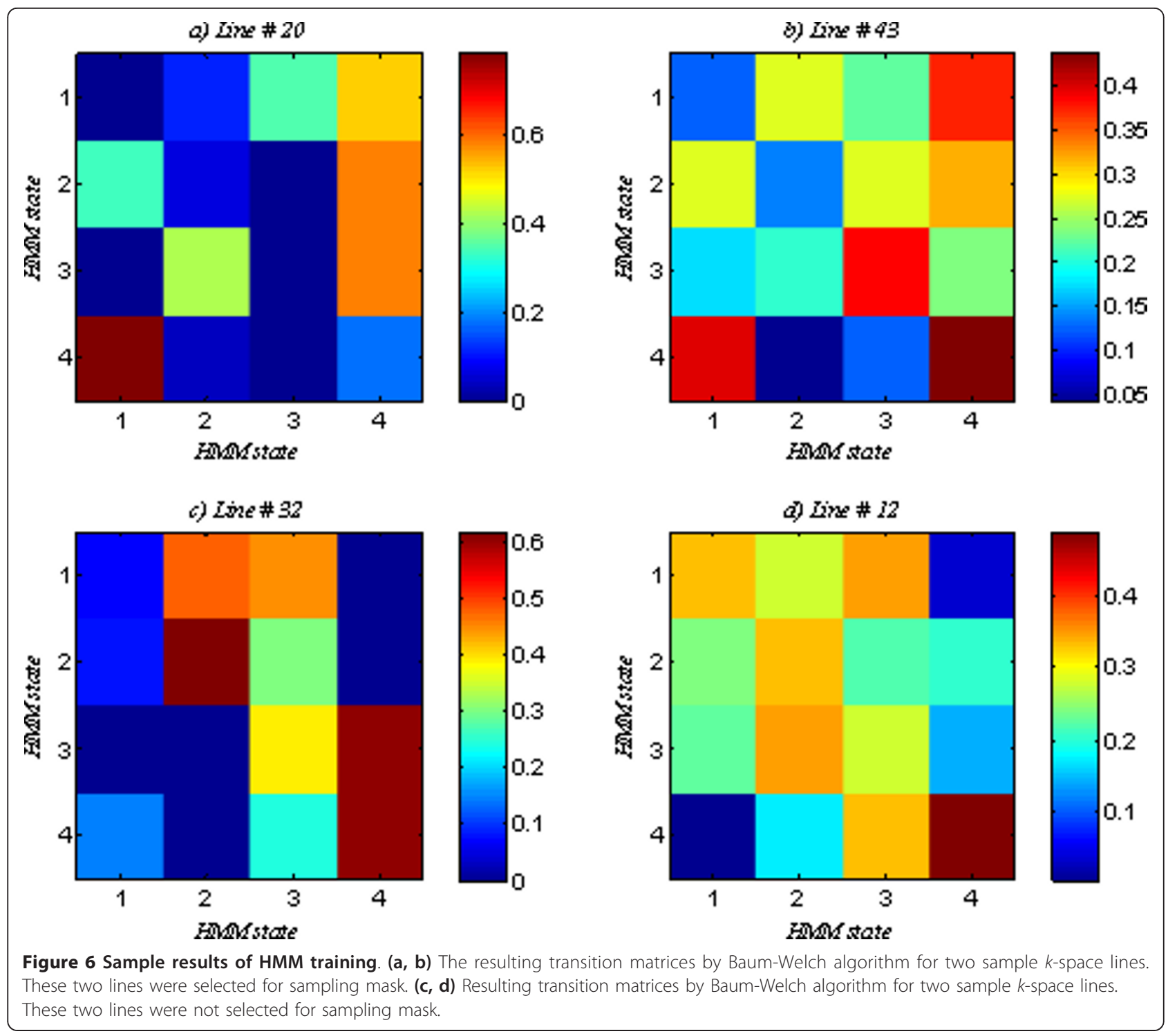

space data to determine the sampling pattern for the other part. We have to consume time for data processing between the two steps of the proposed method. This will add to the overall imaging time and reduce speed of data acquisition to some extent, depending on the computer being used.

\section{Suggestions}

We may use MRI properties to enhance different parts of our algorithm. For example, we do not use the correlation between two adjacent $k$-space trajectories to decide about their selections. Extensions of CS theory, such as distributed CS [11], can be employed to do so. Furthermore, we may adapt our reconstruction algorithm to the characteristics of the MRI data. To this end, we may add MRI-based constraints to the sparsity constraint and reduce reconstruction error or increase the speed and robustness of the reconstruction process.

We may extend our algorithm to non-Cartesian sampling patterns such as spiral and radial sampling [24]. We may apply our proposed method to other types of dynamic MRI such as functional, perfusion, and diffusion imaging.

At present, the proposed iterative thresholding algorithm does not process data gathered by multi-channel coils simultaneously. However, we may extend the proposed method to process such data in parallel. It also does not use data acquired previously from the same subject or other subjects. We may extend the method to use existing data to remove the delay in the data acquisition between the odd and even time frames. This however may degrade quality of the results slightly. 


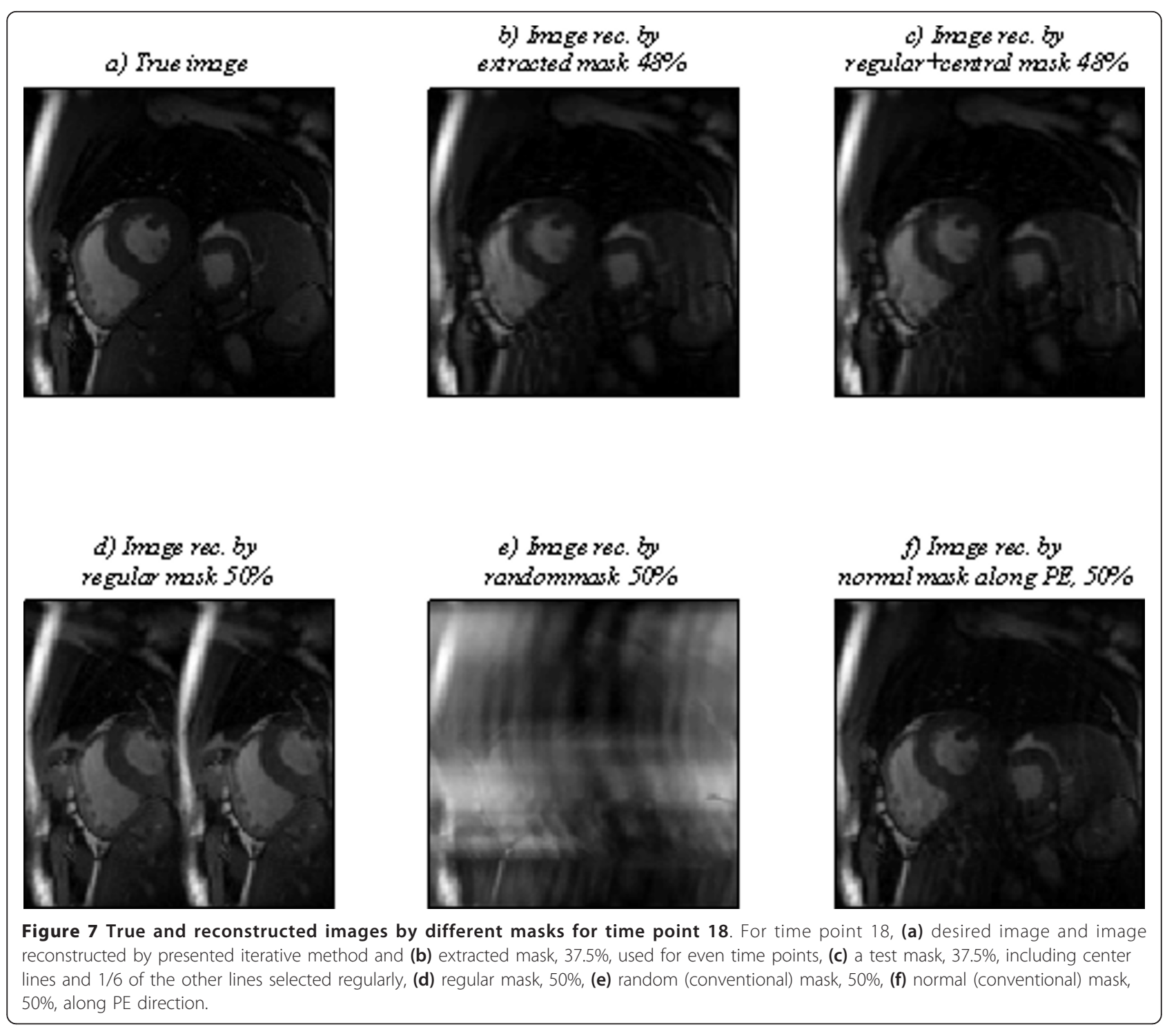

\section{Conclusions}

CS principles can be employed to speed up cardiac cine MRI. In this article, we present a two-step approach to increase the acquisition speed of the cardiac cine MRI by a factor of 2 .

First, we sample the upper half of $k$-space lines for the odd time points of the cardiac cycle. Then, we study the time behavior of $k$-space trajectories by HMM and select the ones that have larger temporal variations to be sampled for the even time points. Fuzzy $c$-means and HMM's are successfully employed as intermediate tools to study time variations of $k$-space trajectories. The sampling pattern extracted from the data is adaptive with the observations (available data) and thus enhances efficiency of the reconstruction.

\section{Methods}

In this article, we present a two-step approach to increase the acquisition speed of the cardiac cine MRI by a factor of 2 . First, we sample the upper half of $k$ space lines, along PE direction, for the odd time points of the cardiac cycle and reconstruct the related images by iterative thresholding. Based on the fact that MRI data are redundant theoretically, we limit our undersampling mask, in both steps of the proposed algorithm, to the upper half of $k$-space. Next, we studied temporal behavior of $k$-space trajectories by HMM and select the ones that have largest variations to be sampled for the even time points. Then, we use iterative thresholding to reconstruct the desired images using the undersampled $k-t$ space data. 


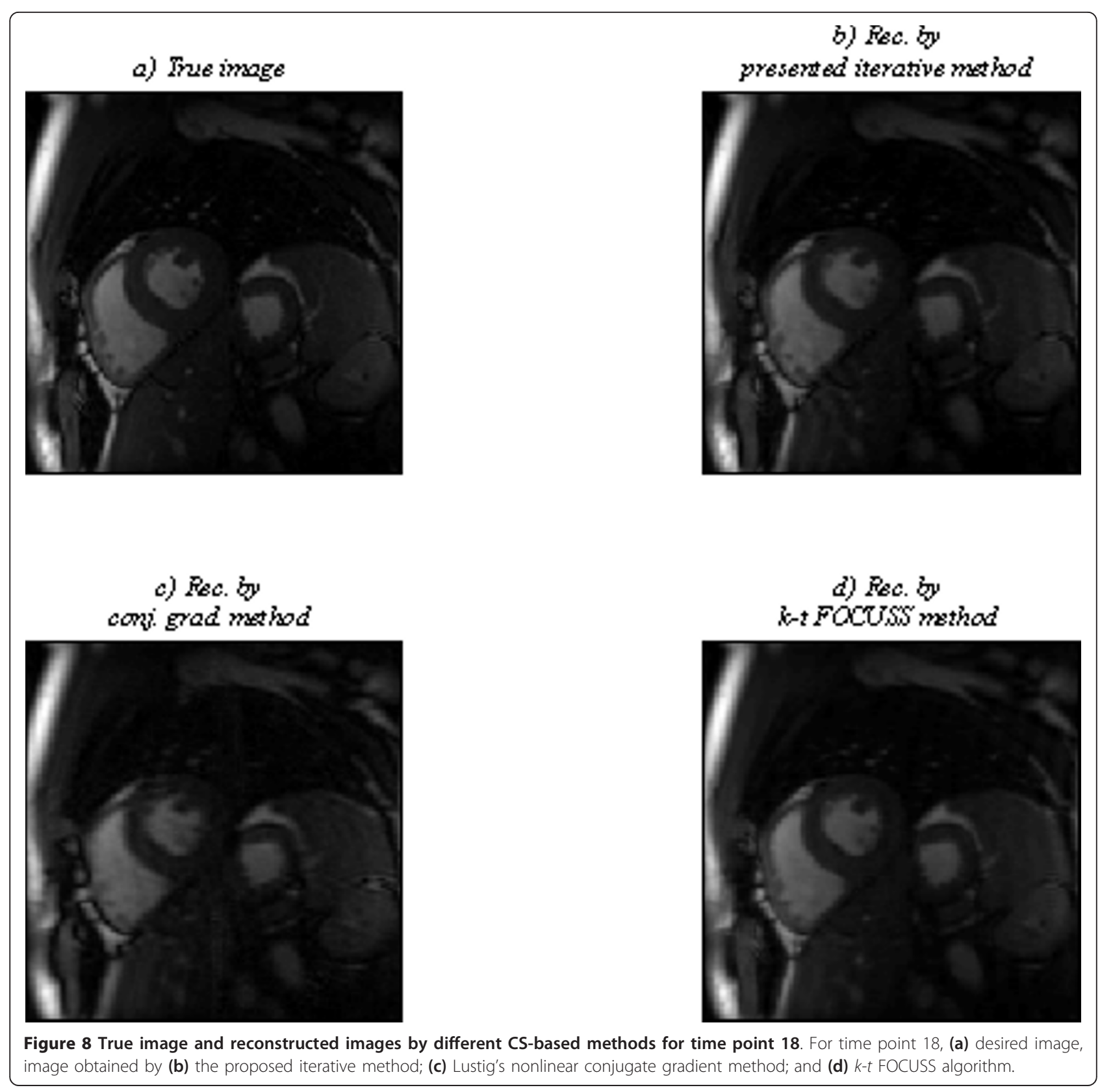

We have successfully applied our proposed method to real ECG gated cardiac cine MRI data with 35 time points. We have changed the matrix size to $128 \times 128$. Main parameters of the dataset are given in Additional file 2.

We have implemented all the algorithms and simulations using MATLAB, R2009a, on a Windows XP based PC with $1.86 \mathrm{GHz}$ centrino processor and $2 \mathrm{~GB}$ RAM. We have also used the codes available at [25] to implement Lustig's nonlinear conjugate gradient methods and [26] to implement $k$ - $t$ FOCUSS algorithm. We have also used the codes available at [27] to implement SENSE and GRAPPA methods.

We have compared the results of the proposed reconstruction method with those of the other methods. Details are described below.

\section{Comparison}

As described in the "Results" section, we have compared performance of our extracted mask with those of four other masks, based on the reconstruction errors of even time points. The reconstruction errors are reported in 


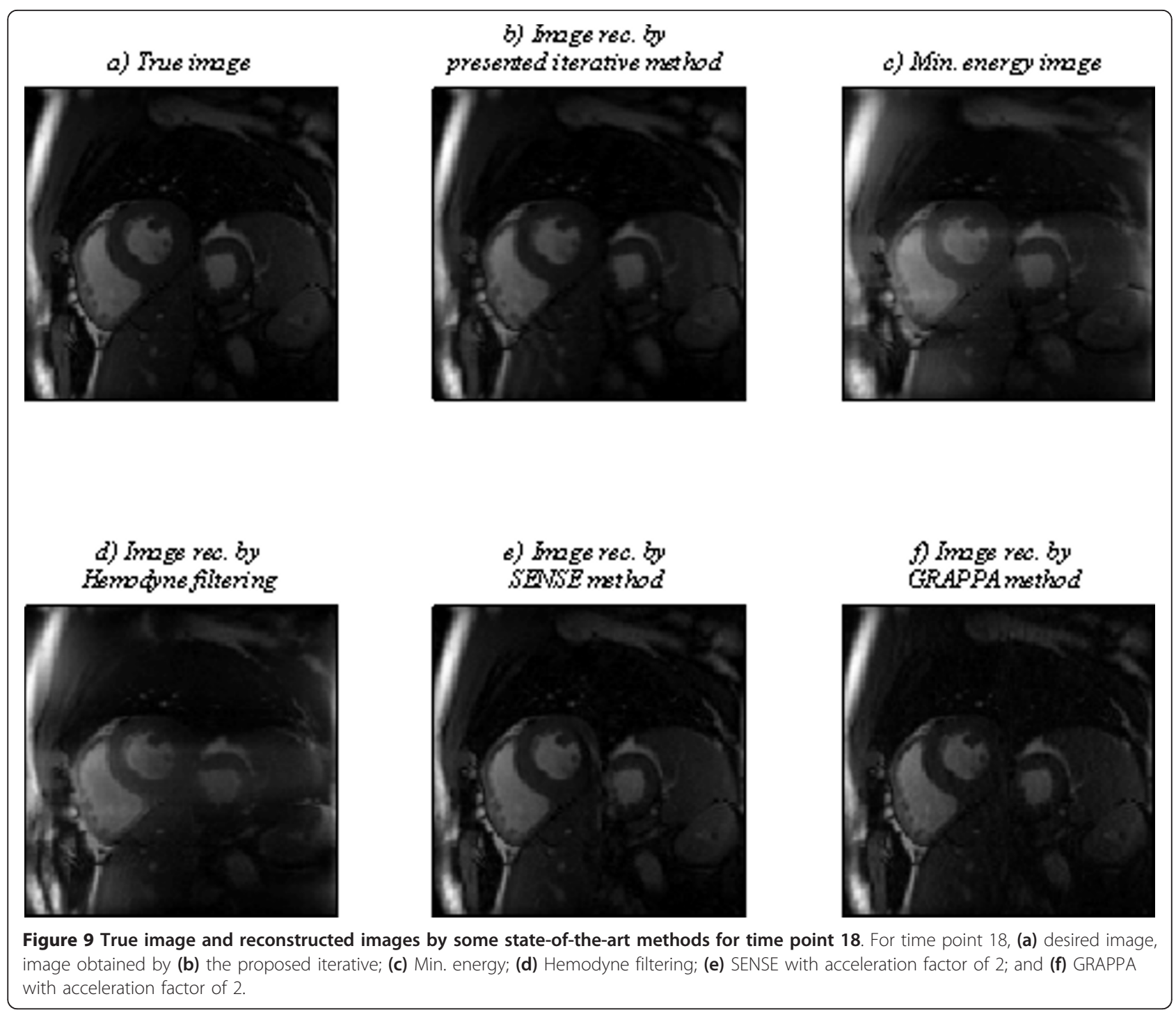

Figure 10. The ranges of relative errors, corresponding to different mask, differ a lot. Thus, we have presented the relative errors in two separate graphs, Figure 10a,b. It can be seen that the proposed mask has the least error.

It must be noted that we have used the following formula to compute relative errors:

$$
\text { Error }=\frac{\| \text { True image }- \text { Reconstructed image } \|^{2}}{\| \text { True image } \|^{2}}
$$

The quality of the images reconstructed by the proposed iterative thresholding method and two other CSbased methods (Lustig's nonlinear conjugate gradient min. of L1 + TV norm and the $k-t$ FOCUSS) are compared in Figure 11a. In Figure 11b, our results are compared with the results of cardiac image reconstruction by SENSE and GRAPPA, two main methods in parallel MRI, with acceleration factor of 2 . It should be noted that the first mask, used for the odd points, includes $62.5 \%$ of $k$-space lines and the second one, used for the even points, includes $37.5 \%$ of $k$-space lines whose corresponding transition matrices are far from the identity matrix. Thus, the overall acquisition time saving is about 50\%. Theoretically, 50\% (the upper half) of $k$-space data is sufficient for reconstructing real MR images. Therefore, our method is compared with the conventional minimum energy and Hemodyne filtering [22], a method based on Hermitian symmetry, and 50\% upper mask along PE direction, in Figure 11c. Our proposed method has the least error in all cases.

Another important parameter that may be used for comparison of the reconstruction methods is the reconstruction time. We have reported the reconstruction times in Table 1. Our method is the third in the list. 

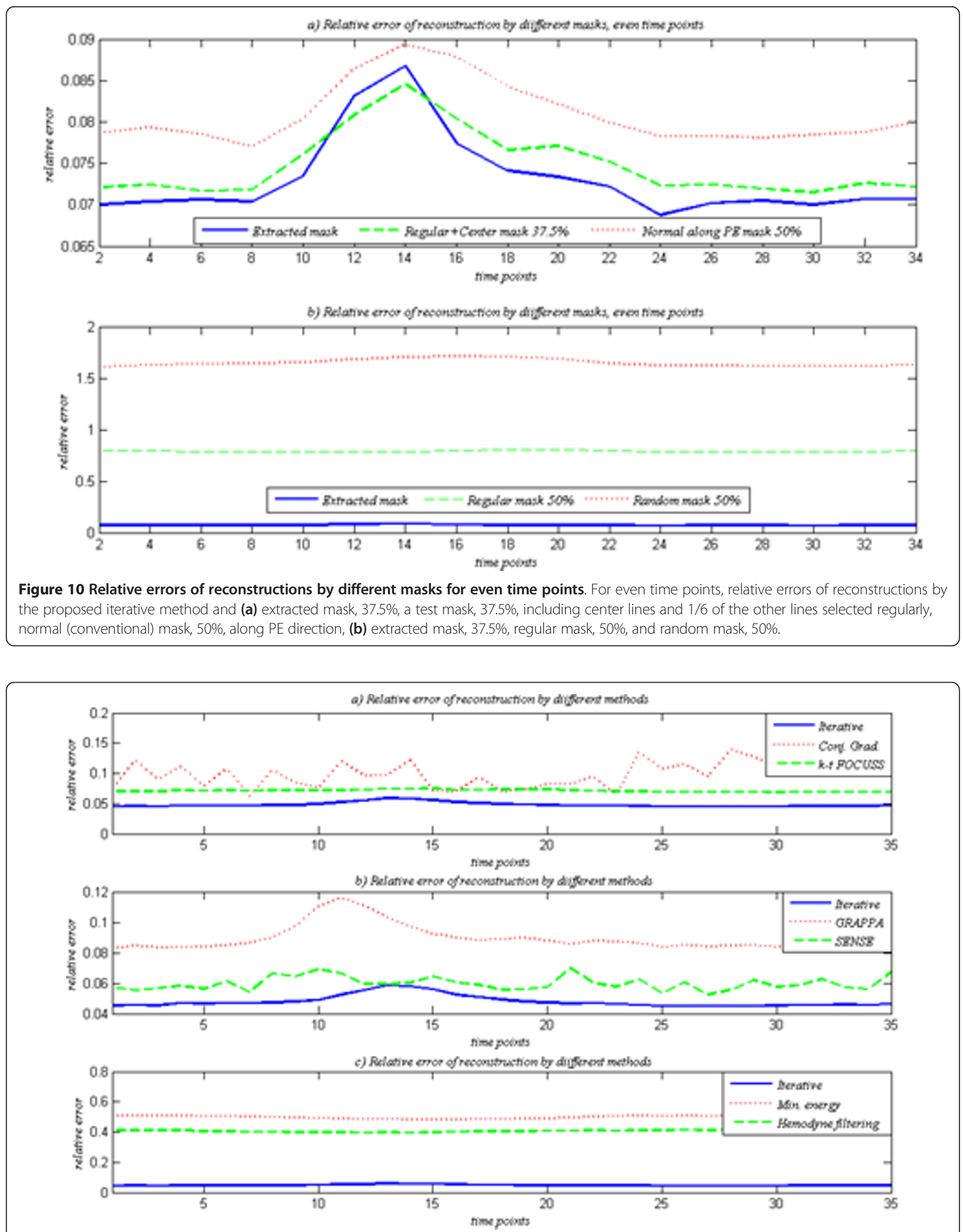

Figure 11 Relative errors of reconstructions by some state-of-the-art methods. Relative error of (a) proposed iterative, Lustig's nonlinear conjugate gradient and $k$ - $t$ FOCUSS, (b) proposed iterative, GRAPPA and SENSE, (c) proposed iterative, minimum energy and hemodyne filtering. 
Table 1 Reconstruction times of different reconstruction methods

\begin{tabular}{ll}
\hline Method & Reconstruction time for each time frame (s) \\
\hline Iterative & 14.6 \\
Conj. Grad. & 604 \\
k-t FOCUSS & 4.8 \\
SENSE & 2.4 \\
GRAPPA & 39.7 \\
\hline
\end{tabular}

Comparison of image reconstruction times of different methods to reconstruct an image corresponding to a time frame.

\section{Additional material}

Additional file 1: Applicability of the proposed method. This file contains scheme, explanations and formulas needed to show how the proposed method can speed up cardiac cine MRI.

Additional file 2: Dataset parameters. This file contains IRB and technical information of used dataset.

\section{Abbreviations}

CS: compressive sensing; DTI: diffusion tensor imaging; fMRI: functional magnetic resonance imaging; HMM: hidden Markov model; MRI: magnetic resonance imaging; PE: phase encoding; SNR: signal-to-noise ratio.

\section{Acknowledgements}

The authors would like to thank Dr. Peter Kellman from Medical Imaging Section Laboratory of Cardiac Energetics at the National Institutes of Health $(\mathrm{NIH})$, Bethesda, MD, USA, for providing the cardiac cine MRI data.

\section{Author details}

${ }^{1}$ Control and Intelligent Processing Center of Excellence (CIPCE), School of Electrical and Computer Engineering, University of Tehran, Tehran 1439957131, Iran ${ }^{2}$ Imaging Research Laboratories, Robarts Research Institute, The University of Western Ontario, 100 Perth Drive, London N6A 5K8, ON Canada ${ }^{3}$ School of Cognitive Sciences, Institute for Research in Fundamental Sciences (IPM), Tehran 1954856316, Iran ${ }^{4}$ Radiology Image Analysis Lab, Henry Ford Health System, Detroit, MI 48202, USA ${ }^{5}$ Department of Radiology, Wayne State University, Detroit, MI 48202, USA

\section{Competing interests}

The authors declare that they have no competing interests.

Received: 16 September 2011 Accepted: 13 April 2012

Published: 13 April 2012

\section{References}

1. J Tsao, P Boesiger, KP Pruessmann, k-t blast and k-t sense: dynamic MRI with high frame rate exploiting spatiotemporal correlations. Magn Reson Med. 50, 1031-1042 (2003)

2. D Sodickson, M Manning, Simultaneous acquisition of spatial harmonics (smash): fast imaging with radiofrequency coil arrays. Magn Reson Med. 38, 591-603 (1997)

3. KP Pruessmann, M Weiger, M Scheidegger, P Boesiger, Sense: sensitivity encoding for fast MRI. Magn Reson Med. 42, 952-962 (1999)

4. B Madore, G Glover, N Pelc, Unaliasing by Fourier-encoding the overlaps using the temporal dimension (unfold), applied to cardiac imaging and fMRI. Magn Reson Med. 42, 813-828 (1999)

5. E Candes, J Romberg, T Tao, Robust uncertainty principles: exact signal reconstruction from highly incomplete frequency information. IEEE Trans Inf Theory. 52, 489-509 (2006)

6. D Donoho, Compressed sensing. IEEE Trans Inf Theory. 54, 1289-1306 (2006)

7. M Lustig, D Donoho, J Pauly, Sparse MRI: the application of compressed sensing for rapid MR imaging. Magn Reson Med. 58, 1182 (2007)
8. I Daubechies, M Defrise, CD Mol, An iterative thresholding algorithm for linear inverse problems with a sparsity constraint. Commun Pure Appl Math. 57, 1413-1457 (2004)

9. U Gamper, P Boesiger, S Kozerke, Compressed sensing in dynamic MRI. Magn Reson Med. 59, 365-373 (2008)

10. M Seeger, H Nickisch, R Pohmann, B Schlkopf, Optimization of $k$-space trajectories for compressed sensing by bayesian experimental design. Magn Reson Med. 63, 116-126 (2010)

11. R Otazo, D Kim, L Axel, DK Sodickson, Combination of compressed sensing and parallel imaging for highly accelerated first-pass cardiac perfusion MRI. Magn Reson Med. 64, 767-776 (2010)

12. M Uecker, Sh Zhang, J Frahm, Nonlinear inverse reconstruction for real-time MRI of the human heart using undersampled radial FLASH. Magn Reson Med. 63, 1456-1462 (2010)

13. M Doneva, P Brnert, H Eggers, C Stehning, J Sénégas, A Mertins, Compressed sensing reconstruction for magnetic resonance parameter mapping. Magn Reson Med. 64, 1114-1120 (2010)

14. H Jung, K Sung, KS Nayak, EY Kim, JC Ye, k-t FOCUSS: a general compressed sensing framework for high resolution dynamic MRI. Magn Reson Med. 61 103-116 (2009)

15. H Jung, J Park, J Yoo, JC Ye, Radial k-t FOCUSS for high-resolution cardiac cine MRI. Magn Reson Med. 63, 68-78 (2010)

16. MH Kayvanrad, Reconstruction from random measurements, in 9th International Conference on Signal Processing, 2008. ICSP 2008, IEEE, 2697-2701 (October 2008)

17. MH Kayvanrad, CA McKenzie, TM Peters, Iterative wavelet thresholding for rapid MRI reconstruction, in SPIE Medical Imaging: Image processing, Lake Buena Vista, Florida, USA, 79624S-1-79624S-10 (2011)

18. M Duarte, M Wakin, R Baraniuk, Wavelet-domain compressive signal reconstruction using a hidden tree model, in ICASSP, Las Vegas, NV, USA, 5173-5140 (2008)

19. R Duda, P Hart, D Stork, Pattern Classification, 2nd edn. (Wiley-Interscience, 2000). ISBN:0471056693

20. $L$ Landweber, An iterative formula for Fredholm integral equations of the first kind. Am J Math. 73, 615-624 (1951)

21. ZP Liang, PC Lauterbur, Principles of Magnetic Resonance Imaging: A Signal Processing Perspective (Piscataway, NJ, IEEE Press, 1999)

22. MA Bernstein, KF Kin, XJ Zhou, Handbook of MRI Pulse Sequences, (Elsevier Academic Press, Burlington, MA 01803, USA, 2004). ISBN: 0-12-092861-2

23. MA Griswold, PM Jakob, RM Heidemann, M Nittka, V Jellus, J Wang, B Kiefer, A Haase, Generalized autocalibrating partially parallel. Magn Reson Med. 47, 1202-1210 (2002)

24. P Zamani, M Kayvanrad, H Soltanian-Zadeh, Cardiac cine MRI using compressive sensing principles, in Proceedings of the 17th Iranian Conference of Biomedical Engineering (ICBME2010), Isfahan, Iran, 3-4 November 2010)

25. http://www.stanford.edul mlustig \SparseMRI.html Accessed 14 Aub 2007

26. http://bisp.kaist.ac.kr/ktFOCUSS.html Accessed 13 Mar 2009

27. http://www.ece.tamu.edu/ mrsl/JIMJI_TAMU/pulsarweb/header.htm Accessed 1 May 2008

doi:10.1186/1687-6180-2012-82

Cite this article as: Zamani et al.: Using learned under-sampling pattern for increasing speed of cardiac cine MRI based on compressive sensing principles. EURASIP Journal on Advances in Signal Processing 2012 2012:82.

\section{Submit your manuscript to a SpringerOpen ${ }^{\circ}$ journal and benefit from:}

- Convenient online submission

- Rigorous peer review

- Immediate publication on acceptance

- Open access: articles freely available online

- High visibility within the field

- Retaining the copyright to your article

Submit your next manuscript at $>$ springeropen.com 